\title{
Pirprofeno* en el Tratamiento de Afecciones Inflamatorias del Tracto Genital
}

\author{
DEPARTAMENTO DE GINECOLOGIA \\ HOSPITAL DE SAN IGNACIO - BOGOTA - COLOMBIA 1987 \\ Dres.: Alberto Angulo Roa** y Víctor Rodríguez R.***
}

\section{INTRODUCCION}

El dolor es probablemente el síntoma más común de las alteraciones ginecológicas y la causa principal de pérdida de horas de trabajo en la mujer.

Diversas causas lo ocasionan, siendo más frecuente la dismenorrea, seguida de los procesos inflamatorios localizados en los órganos del aparato genital, secundarios a infecciones, traumatismos quími$\cos$, radiaciones y tumores (1).

Un avance significativo en la interpretación de la patogenia de estas afecciones fue el descubrir el papel que juegan las prostaglandinas en su desarrollo mediante la disminución del umbral de las terminaciones sensoriales para sustancias dolorígenas como la bradicinina o en forma directa por liberación de las mismas como consecuencia de la distensión y el trauma de la membrana celular. A nivel de inflamación, las prostaglandinas, tromboxanos y lencotrienos con sus acciones fisiológicas complejas, intervienen de manera importante ya que por ser lencotácticas, sostienen la exudación celular (3).

\footnotetext{
* RENGASIL (Ciba-Geigy)

** Profesor Departamento de Ginecología. Hospital San Ignacio. Presidente Sociedad Colombiana de Obstetricia y Ginecología. *** Profesor Departamento de Ginecología. Hospital San Ignacio. Vicepresidente Sociedad Colombiana de Obstetricia y Ginecología.
}

La elevación entonces de las prostaglandinas, es la causa directa de la inflamación y el dolor (3, $5)$, síntomas estos que requieren la atención del médico y de una planificación terapéutica enfocada a la utilización de medicamentos que inhiban la síntesis de estas sustancias.

En 1971 Vane, descubrió que los antiinflamatorios no esteroides influian en la síntesis de prostaglandinas. La inhibición de actividad de sintetasa de prostaglandina a nivel de un foco inflamatorio, explica el efecto de estas drogas sumadas a otras acciones que contribuyen a su actividad inflamatoria, tales como: inhibición de la plasimina, por tanto disminución en la formación de cinina; inhibición de la hialuronidasa que origina un menor aumento de presión osmótica del exudado inflamatorio; inhibición de la migración y la actividad fagocítica de las células retículo endoteliales y leucocitos polimorfo nucleares, originándose una menor acumulación de células que proporcionan sintetasa de prostaglandinas, después de destruidas por el linosomar. La inhibición de la agregación plaquetaria reduce además la liberación de renotonina y compuestos adenilícos $(6,8)$.

En los últimos años se ha incrementado la investigación para desarrollar nuevos antiinflamatorios no esteroides que sean más fáciles, mejor tolerados y de cómoda posología (9). Un ejemplo concreto son los derivados del ácido propiónico y entre ellos el pirprofeno, Rengasil, que cuenta con un amplio margen terapéutico $(10,11)$. 
Más del $80 \%$ de la cantidad total absorbida penetra en la circulación 1 hora después de ingerirse las cápsulas. La biodisponibilidad alcanza un nivel cercano al $100 \%$. Su metabolismo se completa esencialmente en 24 horas, recuperándose en la orina menos del $1 \%$ de la droga inalterada. La vida media es aproximadamente de 7 horas.

Los estudios farmacológicos realizados por Maier y Col. (12), demuestran la superioridad antiinflamatoria de Rengasil ( $\mathrm{r}$ ) frente al ácido acetil salicílico ( 80 veces), el ibuprofen ( 10 veces), y el sulindac (3).

Su índice terapéutico resultó más favorable que el de los demás antiinflamatorios no esteroides mencionados anteriormente, lo cual indica un buen margen de seguridad desde el punto de vista de la tolerabilidad.

Ha sido ampliamente utilizado para el tratamiento del dolor y la inflamación en las afecciones del aparato locomotor con resultados bien satisfactorios $(8,11)$. Con base en los anteriores criterios, se llevó a cabo este estudio, con el fin de corroborar la eficacia y tolerabilidad de Rengasil cápsulas de $400 \mathrm{mg}$ en el manejo de los trastornos músculo esqueléticos de diversa etiología.

\section{MATERIAL Y METODOS}

Se realizó un estudio abierto no comparativo de tipo prospectivo longitudinal en 40 mujeres voluntarias mayores de 18 años, con afecciones dolorosas del aparato genital posibles de ser tratadas en forma ambulatoria con RENGASIL, siempre y cuando no estuvieran en embarazo o lactando, o con terapia anticoagulante. Con antecedentes o síntomas de úlcera gastroduodenal activa o recurrente, colitis ulcerosa, insuficiencia cardíaca descompensada o hipertensión grave; con hallazgos de laboratorio anormales que en opinión del investigador justificaran la exclusión del ensayo, especialmente aquellos que indiquen una afección hepática o renal grave o trastornos hematológicos. También se excluyeron pacientes con diabetes mellitus manifiesta, trastornos metabólicos (aparte de los que puedan ser atribuidos a la artritis reumatoidea), hipersensibilidad a cualquiera de los componentes del medicamento en estudio, como también pacientes con tratamiento durante las tres semanas precedentes con inmunosupresores, D-penicilina, cloroquina, ACTH, corticosteroides (tratamiento local o sistémico) y sindrome de mala absorción.

Todas las pacientes recibieron una cápsula de RENGASIL $400 \mathrm{mg}$ dos veces al día hasta un máximo de dos semanas consecutivas. Si la curación se producía antes de las dos semanas, se daba por terminada la prueba en ese momento.

Se llenó un formulario de historia clínica en donde se consignaron las variables referentes al estudio como nombre, edad, peso, talla, diagnóstico, tratamientos concomitantes, efectos secundarios, duración del tratamiento, eficacia de la terapia $(1=$ nula, $2=$ mínima, $3=$ suficiente, y $4=$ excelente). La tolerabilidad se evaluó según los efectos secundarios atribuibles al medicamento $(1=$ muy pobre, 2 = pobre, $3=$ buena, y $4=$ excelente), el comienzo de la acción en horas o minutos, interrupción del tratamiento y sus causas $(1=$ curación, 2 = efectos secundarios, $3=$ ineficacia de la droga, y $4=$ otros).

No se permitieron tratamientos concomitantes de tipo analgésico o antiinflamatorio, pero sí las medicaciones que eventualmente estuviera recibiendo para el tratamiento de otras afecciones, siempre y cuando no se modificaran las dosis.

\section{RESULTADOS}

Se estudiaron 40 mujeres con una edad promedio de $29+7$ años, peso promedio de $55+7 \mathrm{Kg}$. talla promedio de $159+6 \mathrm{~cm}$, (Tabla 1$)$, con afecciones dolorosas del aparato genital, siendo las más frecuentes las EPI en el $57.5 \%(\mathrm{n}=23)$. Le siguen la anexitis $(n=9,22.5 \%)$, y la dismenorrea $(n=6,15 \%)$, (Tabla 2).

El tiempo de evolución de las afecciones en el momento de la consulta fue para la anexitis de 4 días; 3 días para la EPI y de 2 días para la dismenorrea.

Todas las pacientes con diagnóstico de EPI recibieron terapia antibiótica concomitante. Tres de las pacientes con anexitis también recibieron anti- 
TABLA 1

\begin{tabular}{|c|c|c|c|c|}
\hline Pacientes & \multicolumn{4}{|c|}{40 Mujeres } \\
\hline Edad (Años) & $X=29$ & $\mathrm{SD}$ & & 7 \\
\hline Rango & 18 & & 55 & \\
\hline Peso (kilos) & $X=55$ & SD & & 7 \\
\hline Rango & 45 & & 85 & \\
\hline Talla (CMS) & $X=159$ & $\mathrm{SD}$ & $=$ & 6 \\
\hline Rango & 145 & & 172 & \\
\hline Duración de la & & & & \\
\hline Afección (días) & $\mathrm{X}=$ & $\mathrm{SD}$ & & 2 \\
\hline Rango & 1 & & 30 & \\
\hline Comienzo & & & & \\
\hline Analgesia & $X=1: 30$ & $\mathrm{SD}$ & $=$ & 30 \\
\hline
\end{tabular}

TABLA 2

DISTRIBUCION PORCENTUAL DE MUJERES SEGUN DIAGNOSTICO DE INGRESO

\begin{tabular}{|lrr|}
\hline \multicolumn{1}{|c|}{ Diagnóstico } & N & $\%$ \\
\hline EPI & 22 & 55.0 \\
Anexitis & 9 & 22.5 \\
Dismenorrea & 5 & 12.5 \\
EPI y Aborto Séptico & 1 & 2.5 \\
Hidrosalpinx & 1 & 2.5 \\
Osteitis Pubis vs. & 1 & 2.5 \\
Hematoma Retzius & 1 & 2.5 \\
No Especificado & 1 & \\
\hline
\end{tabular}

bióticos y el resto no recibieron ningún otro medicamento diferente a Rengasil (R).

Para el grupo de pacientes con EPI el tiempo de duración del tratamiento fue de 9 días al igual que para la anexitis pero estas dos entidades frente a la dismenorrea presentan significancia estadística, en cuanto a la duración del tratamiento $(\mathrm{p}<0,01)$.

$\mathrm{Al}$ inicio de la acción analgésica fue de 1:30 minutos promedio para las pacientes con anexitis y dismenorrea, y de 2 horas para las enfermas con EPI con diferencia significativa entre este último diagnóstico y los dos anteriores $(p<0,01)$. En resumen en los dos grupos el $81.6 \%$ de los casos la acción analgésica estuvo entre 1 y 2 horas (Figura 1 ).

FIGURA 1

\section{COMIENZO DE LA ACGION ANALGESICO ANTIINFLAMATORIA}

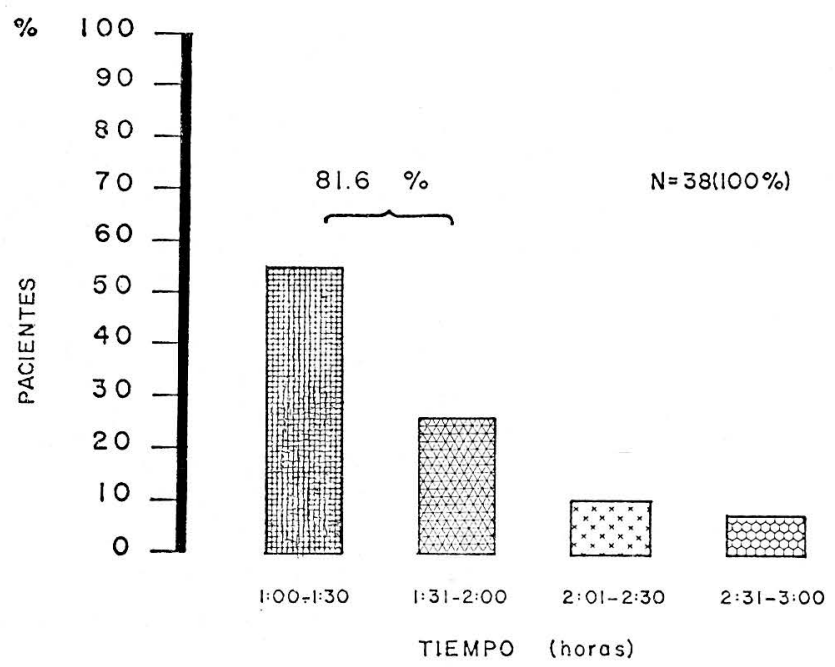

La evaluación del efecto terapéutico con resultados excelentes fue del ciento por ciento para todos los casos de dismenorrea; para la anexitis del $89 \%$ y en las pacientes con EPI del 70\%. Dos casos de este último diagnóstico tuvieron respuesta mínima o regular. Globalmente la respuesta terapéutica fue excelente en el $75 \%$ de los casos y buena en el $25 \%$. Hay diferencia estadísticamente significativa en la respuesta terapéutica en las pacientes con dismenorrea y anexitis. Respecto a aquellas con diagnóstico de EPI resultados consecuentes con la fisiopatología y clínica de estos procesos, (Figura 2).

La tolerabilidad se comporta de igual forma al realizar el análisis entre los grupos y globalmente fue excelente y buena en el $87.5 \%$ de los casos.

Es de anotar que todas las pacientes del grupo de dismenorrea no presentaron ningún efecto secundario y este grupo no recibió ninguna medicación concomitante. 
FIGURA 2

\section{EFECTO TERAPEUTICO: EVALUACION GLOBAL}

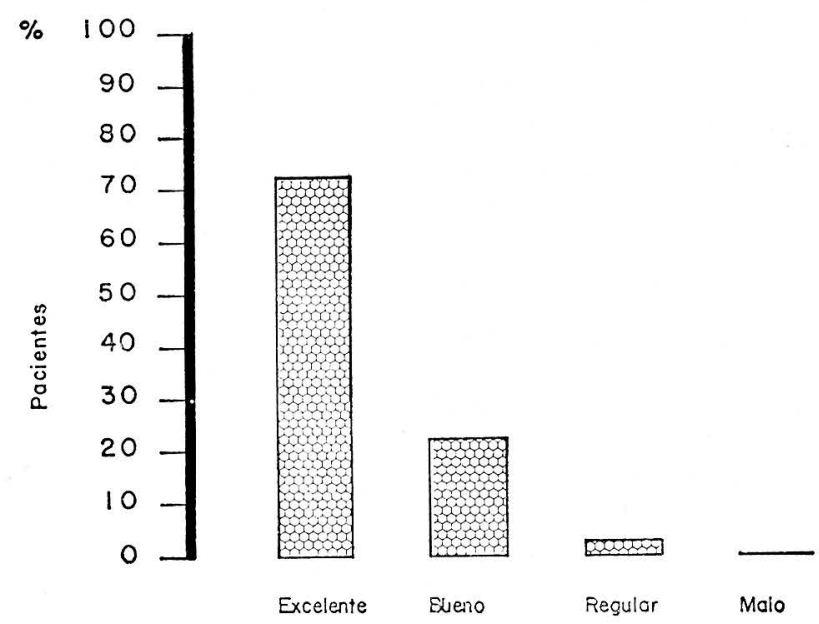

En el grupo de las pacientes con diagnóstico de anexitis, dos de ellas presentaron epigastralgia y cefalea. Estas pacientes recibían concomitantemente tratamiento con antibiótico oral en el primer caso y parenteral en el segundo; por este motivo no se puede atribuir la reacción adversa asociada, al Rengasil.

El $17.5 \%(\mathrm{n}=7)$ de las pacientes con diagnóstico de EPI presentaron epigastralgia, náuseas y cefalea. Todas ellas recibían terapia antibiótica concomitante de tal manera que no se puede determinar asociación entre el efecto secundario y la ingestión de Rengasil.

\section{DISCUSION}

Rengasil ha demostrado a través de numerosos estudios clínicos su eficacia y seguridad en el manejo de las patologías asociadas con procesos inflamatorios $(3,10)$ tanto crónicos como agudos. Hay un creciente número de estudios clínicos sobre la acción de este medicamento en los procesos inflamatorios del aparato genital $(13,17,22)$, así como estudios comparativos con otros AINES $(13,21)$ que demuestran la superioridad del pirprofeno en estas indicaciones.

Los resultados obtenidos en las pacientes que participaron en el estudio, indican que la valoración global del efecto terapéutico fue excelente $75 \%$ y buena en el $22.5 \%$. Al igual que de los casos y la tolerabilidad en el $87.5 \%$. Es de resaltar que los efectos varios que se reportaron en el $25 \%$ de los casos no son atribuibles plenamente al Rengasil y fueron de carácter leve sin propiciar la interrupción del $4 \%$.

En todas las pacientes se administraba concomitantemente terapia antibiótica y se resalta la ausencia de efectos colaterales en las pacientes con diagnóstico de dismenorrea y que no recibieron ningún otro medicamento diferente al del motivo del estudio.

Dada la importancia que juega el dolor en los procesos inflamatorios ginecológicos, se hace hincapié en la rapidez del alivio experimentado por las pacientes, dado que el promedio en el inicio de acción analgésica fue de una hora y media en el $82 \%$ de los casos.

El tiempo promedio de duración de la afección fue del $5+1-2$ días en el $90 \%$ de las pacientes y la duración del tratamiento tuvo un promedio de $8+1-2$ días.

Se corroboran mediante este estudio los hallazgos de otros investigadores y se demuestra la eficacia de Rengasil como coadyuvante en el tratamiento de los procesos inflamatorios del área ginecológica.

Composición: Pirprofeno: cápsulas de $400 \mathrm{mg}$ : ampollas de $400 \mathrm{mg} / 4 \mathrm{ml}$ Indicaciones: Antiinflamatorio Analgésico. Inflamaciones agudas que cursan con dolor de cualquier etiología, especialmente las relacionadas con ORL (amigdalitis, faringitis, otitis), con trauma (esquinces, luxaciones, fracturas), en lumbalgia y en estados inflamatorios/dolorosos en ginecología (anexitis, dismenorrea primaria), ataque agudo de gota. Dosificación: Adultos (vía oral), una cápsula de $400 \mathrm{mg}$ dos veces al día; en caso necesario puede aumentarse a 3 veces diarias (el tope máximo en la dosis es de $1.200 \mathrm{mg} /$ día). Vía parenteral: una ampolla diaria de $400 \mathrm{mg}$ por vía intraglútea profunda, en el cuadrante superior externo. Si es necesario puede aplicarse una segunda ampolla al cabo de varias horas en el otro glúteo y durante máximo 5 días. Contraindicaciones: Ulcera péptica, afección hepática;, hipersensibilidad conocida a la sustancia activa, asmáticos con hipersensibilidad previa al ácido acetilsalićlico u otros medicamentos que inhiben la prostaglandina-sintetasa. Precauciones: Antecedentes de afecciones gastrointestinales, menoscabo grave de la función renal o cardiaca y embarazo. Tratamiento prolongado: vigílese la función hepática. No es recomendable para niños menores de 14 años. Efectos secundarios: Raramente: hemorragias gastrointestinales, úlcera péptica, anomalías de la función hepática y renal, reacciones de hipersensibilidad. Presentaciones: Caja con 12 cápsulas de $400 \mathrm{mg}$ y caja con 5 ampollas de $400 \mathrm{mg} / 4 \mathrm{ml}$. (Registro M-006786 y M-006991 M.S.).

Para mayor información favor consultar el Departamento Médico de CIBA-GEIGY Colombiana S.A. Apartado 12323, Teléfono 2612277 Bogotá - Colombia. 


\section{BIBLIOGRAFIA}

1. NOVAK E. Tratado de Ginecología 8a. ed. 1982.

2. BOWMAN y RAND. Farmacología láser bioquímicas y patológicas 2da. Ed. 1984; 13: 13-15.

3. GIMENO M. Prostaglandinas y Endocrinología. La semana médica 1979; 8: 154 .

4. KAVPPILA. A. et al. Prostaglandin biosyntesis inhibitors and endometriosis. Prostaglandins, 1979, 18: 655.

5. ANDERSON A.B.M. HAYNES, P. Reduction of menstrual blood loss by prostaglandin syntetase inhibitors. Lancet 1976 774: 1-10.

6. MURRAY, W. Non steroical anti-inflamatory agents, Annual Review of Pharmacology 1970; 98: 10-171.

7. SPECTOR. W.G. The pharmacology of inflammation English University Press London, 1968.

8. ZWEIFACH, B. The inflammatory process. Academic Press, London, 1974.

9. BARRY, S. Tratamiento farmacológico del dolor, 1983.

10. MAIER R. Aspectos farmacológicos del Pirprofeno, 1982.

11. MAIER, R. Aspectos farmacológicos del Pirprofeno proceedings of an Internacional Symposium on pirprofeno held in Moscow on 18th June 1983 during the European Congress of Rheumatology.

12. CHRT, J.J. Reseña de la farmacología preclínica del pirprofeno en Van des Rost.
13. YOUNIS MN. An evaluation of pirprofeno in the treatment of primary dysmenorrhea. Departament of obstetrics and gynecology Faculty of medicine al Azhar University al Hussein Cairo - Egypl.

14. CHAN et al. Prostaglandin in primary dysmenorrhea Comparison of prophylactic and non prophylactic treatment with Ibuprofen and use of oral contraceptives. Am. J. Med. 1981, 70: 535 .

15. CORSON, S.L. BOLOGNESE, R.J. Ibuprofen therapy for dysmenorrhea J. Reprod Med. 1979: 20: 246.

16. LARKIN. R.M. Dysmenorrhea treatment with antiprostaglandin. Obstet. and Gynec. 1979; 456: 54.

17. MENDEZ, F.J. Pirprofeno oral en dismenorrea primaria. Compendium de Investigaciones. Clínicas Latinoamericanas 1985; V: 1 .

18. SERENO, J.A. Estudio comparativo de Piroxicam y aido mefeniamico en el tratamiento de la dismenorrea. Investigación Médica Internacional, 1985; 12 : 3.

19. BAStos, A.C., BAGnOLI, V.R. Piroxicam en dismenorrea. Rev. Bras. Clin. Terap. 1984; 13 (6): 234.

20. FRIEDRICH, E.A. A comparison of Etrilolac with aspirin and placebo in patients with episiotomy pain Cur Ther Rev. 1983: $33,100$.

21. BLOOMFIELD, S.B. et al. A comparison of pirprofen, aspirin, acetaminophen, and placebo in post partum uterine cramp pain current therapy. Rev. 301981 ; 139: 145 .

22. ELSNER, C.W. et al. Pirprofen in dismenorrhea, A. double blind placebo controlled trial. Clin, pharmacol. 1980; 27 (2): 251-52. 